\title{
Nucléaire
}

de Lyon

\section{Dark matter search with a low temperature sapphire bolometer}

A. de Bellefon ${ }^{1}$, I. Berkes ${ }^{2}$, C. Bobin' ${ }^{2}$, D. Broszkiewicz ${ }^{1}$, B. Chambon ${ }^{2}$, M. Chapellier ${ }^{3}$, G. Chardin ${ }^{4}$, P. Charvin ${ }^{5}$, V. Chazal ${ }^{2}$, N. Coron ${ }^{8}$,

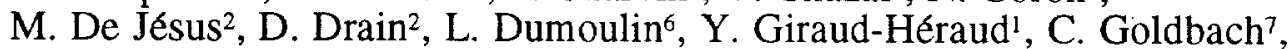
G. Guerier ${ }^{3}$, J.P. Hadjout ${ }^{2}$, J. Leblanc ${ }^{8}$, D. Marchand ${ }^{1}$, M. Massaq ${ }^{2}$, Y. Messous ${ }^{2}$, X. Navick 3 , G. Nollez, P. Pari ${ }^{3}$, C. Pastor ${ }^{2}$, M.C. Perillo-Isaac 1 , I. Prostakov ${ }^{4}$, D. Yvon ${ }^{4}$

${ }^{1}$ Laboratoire de Physique Corpusculaire, Collège de France, F-75231 Paris Cedex 05 ${ }^{2}$ Institut de Physique Nucléaire de Lyon, IN2P3/CNRS, Université Claude Bernard, F-69622 Villeurbanne Cedex, France

${ }^{3}$ CEA, Centre d'Etudes de Saclay, DRECAM, F-91191 Gif-sur-Yvette Cedex

${ }^{4} C E A$, Centre d'Etudes de Saclay, DAPNIA, F-91191 Gif-sur-Yvette Cedex

${ }^{5}$ Laboratoire Souterrain de Modane, CEA-CNRS, F-73500 Modane

${ }^{6}$ CSNSM, IN2P3-CNRS, Université Paris XI, F-91405 Orsay Cedex

${ }^{7}$ Institut d'Astrophysique de Paris, CNRS, F-75014 Paris

${ }^{8}$ Institut d'Astrophysique Spatiale, Univ. Paris XI, F-91405 Orsay Cedex

EDELWEISS Collaboration

MANIOLA Project

to be published in Astroparticle Physics 


\section{Accefti à Astrotarticle Physics (1996)}

Dark matter search

with a low temperature sapphire bolometer
A. de Bellefon ${ }^{1, \dagger}$, I. Berkes ${ }^{2}, \dagger$, C. Bobin ${ }^{2}, \dagger$, D. Broszkiewicz ${ }^{1, \dagger}$, B. Chambon ${ }^{2, \dagger}$,

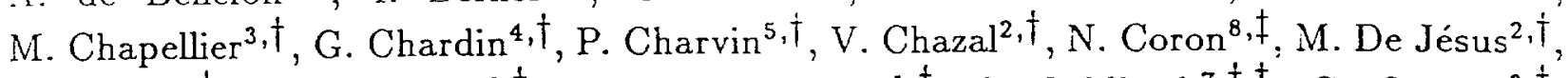 D. Drain ${ }^{2, \dagger}$, L. Dumoulin ${ }^{6, \dagger}$, Y. Giraud-Héraud ${ }^{1, \dagger}$, C. Goldbach ${ }^{7, \dagger}$, G. Guerier ${ }^{3}, \dagger$,

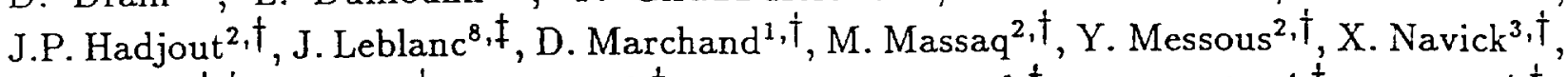

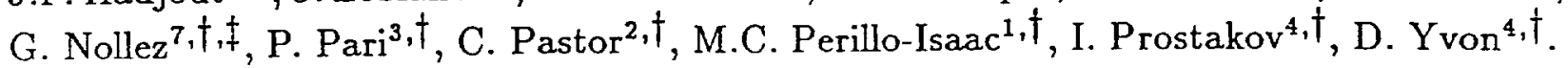

1 Laboratoire de Physique Corpusculaire, Collège de France, 11 place M. Berthelot, F-75231 Paris Cedex 05, France.

2 Institut de Physique Nucléaire de Lyon and Université Claude Bernard, Lyon I, IN2P3-CNRS, 43 Bd du 11 novembre 1918, F-69622 Villeurbanne Cedex, France.

3 CEA, Centre d'Etudes de Saclay, DRECAM, Service de Physique de l'Etat Condensé, F-g1191 Gif-sur-Yvette Cedex, France.

${ }^{4}$ CEA, Centre d'Etudes de Saclay, DAPNIA, Service de Physique des Particules, F-91191 Gif -sur-Yvette Cedex; France.

5 Laboratoire Souterrain de Modane, CEA-CNRS, 90 rue de Polset, F-73500 Modane, France

${ }^{6}$ Centre de Spectrométrie Nucléaire et de Spectrométrie de Masse, IN2P3-CNRS, Université Paris XI, Bat.108, F-91405 Orsay Cedex, France.

7 Institut d'Astrophysique de Paris, CNRS, 98 $8^{\text {bis }}$ boulevard Arago, F-75014 Paris, France.

${ }^{8}$ Institut d'Astrophysique Spatiale, Université Paris XI, Bat. 121, F-91405 Orsay Cedex, France

† EDELWEISS Collaboration

$\div$ MANOLIA Project 
Abstract

A dark matter detection experiment using a low temperature $24 \mathrm{~g}$ sapphire bolometer is presented. The low radioactive background cryogenic facility, installed in a deep underground site is described, as well as the low-noise read-out electronics and the data analysis. From the energy spectrum, measured down to $4 \mathrm{keV}$, exclusion plots are derived for WIMPs having coherent vector coupling or axial coupling to ordinary matter.

PACS code: $95.35+\mathrm{d}$

Keywords: Dark Matter, WIMP, Bolometer 


\section{Introduction}

The quest for dark matter is at the centre of observational projects and of direct detection experiments as well as of cosmological search. Observations dedicated to the detection of baryonic dark matter in the form of dark or faint objects, and based on the microlensing effect, are performed by several groups [1-4]. Analyses of the first results report a fraction of massive compact objects in the Galactic halo lower than $30-35 \%[5,6]$. In addition, new determinations of the primordial deuterium abundance $[7,8]$ lead to a low value of the baryonic density of the universe, leaving little room for baryonic matter in the extended halos of elliptical galaxies [9]. Direct detection experiments of non-baryonic dark matter, in the form of Weakly Interactive Massive Particles (WIMPs) appear then necessary. Among these particles, the most prominent candidate is the lightest spin-1/2 neutral supersymmetric particle $[10,11]$.

Goodman and Witten [12] proposed in 1985 to detect WIMPs through their elastic scattering on nuclei giving rise to experiments using semiconductor or scintillator detectors (see for instance [13] for a recent review and references therein) as well as to experimental projects using bolometers. Bolometers, operated at low temperature $(<50 \mathrm{mK})$, can reach very low energy detection threshold and resolution $(<\mathrm{keV})$ with a full energy conversion for any recoiling particle, and appear as particularly promising. Moreover, it has been experimentally shown that the simultaneous measurement of heat and another form of deposited energy (ionisation, light) leads to the possibility of background rejection (see 14? for a recent overview of the field). The main challenges are now to achieve a very low radioactive background in a cryogenic environment and to maintain a low detection threshold for massive detectors $(\sim 1 \mathrm{~kg})$. 
In this context, the EDELWEISS collaboration (Expérience pour DEtecter Les WImps En Site Souterrain) designed a deep underground low radioactivity cryogenic facility devoted to WIMPs bolometric detection at the Laboratoire Suterrain de Modane (LSM). The present status of the installation and first results obtained with a small size $(24 \mathrm{~g})$ sapphire bolometer (MANOLIA realisation) are presented here.

\section{Experimental set-up}

\subsection{The Laboratoire Souterrain de Modane}

The LSM is situated in the Alps at the French-Italian border, in the Fréjus highway tunnel. The site offers a $1780 \mathrm{~m}$ rock coverage against cosmic radiation, mainly constituted of lustrated schists ( $4800 \mathrm{~m}$ water equivalent). The measured residual cosmic muon flux is of $\sim 4 \mathrm{~m}^{-2}$.day ${ }^{-1}$, i.e. an attenuation of the surface flux by a factor of $\sim 10^{6}$. The fast neutrons flux (energy between 1 and $5 \mathrm{MeV}$ ), recently measured, is $\sim 180 \mathrm{~m}^{-2}$. day ${ }^{-1}$ [15]. Under normal air renewal (complete change of the lab volume twice every hour) the long time average of the radon concentration is $15 \mathrm{~Bq} \cdot \mathrm{m}^{-3}$.

\subsection{Cryogenics and shielding}

The home-made dilution refrigerator is built with selected low-radioactivity materials. The upper part (cryogenic fluid tanks, pumping tubes) is built with stainless steel with low content of cobalt (activity $<0.03 \mathrm{~Bq} \cdot \mathrm{kg}^{-1}$ ). The lower part (vacuum jacket and radiation shields enclosing the dilution unit) is made of selected copper. The only measurable activity $\left(0.07 \mathrm{~Bq} \cdot \mathrm{kg}^{-1}\right)$ comes from the few grams of silver powder used in the heat exchangers and the mixing chamber (thermal neutron activation isotope ${ }^{110 \mathrm{~m}} \mathrm{Ag}$ ). The base temperature of the dilution refrigerator with complete wiring is $35 \mathrm{mK}$, with a circulation rate of 
$20 \mu \mathrm{mol} . \mathrm{s}^{-1}$. The available experimental volume is a cylinder, $80 \mathrm{~mm}$ in diameter and $300 \mathrm{~mm}$ in height. An autonomy of two weeks for liquid nitrogen and five days for helium is achieved, allowing continuous day-long experimental runs. An automated gas handling system has also been developed. It greatly simplifies the cooling and the maintenance of the cryostat and allows a remote control of the experiment.

Great care is taken for the isolation of the cryostat from microphonic noise due to ground vibrations, and from natural radioactivity of the lab walls. An antisismic platform, lying on 4 pneumatic isolators (pressure 5 bars), acts as a low-pass filter ( $\geq 3 \mathrm{~Hz}$ ) and tolerates a 20 tons load. Enclosing the refrigerator, an outer shield is made of selected low-radioactivity materials, including in sequence from the outside: phonic absorbent, steel layer $(5 \mathrm{~cm}$, also used for mechanical sustainment), air-tight plastic wall (for $\mathrm{N}_{2}$ flushing and radon removal), lead $(10$ to $15 \mathrm{~cm})$ and copper $(10 \mathrm{~cm})$, see fig. 1.

In the cold part of the dilution unit, within the experimental volume, a close shield of roman-time lead provides a $4 \pi$ steradians shielding $(2-9 \mathrm{~cm}$ thickness $)$ around the detector, see fig.2. Most of the components entering this ultimate shield are selected to a radioactivity level below the background level of the LSM $100 \mathrm{~cm}^{3}$ germanium detectors.

\subsection{Detector and low-noise read-out}

The bolometer is a $24 \mathrm{~g}$ sapphire cylinder equipped with a NTD germanium thermistor. This detector has been extensively investigated previously: for a detailed description of the bolometer and its performances, in particular the energy resolution from $5.89 \mathrm{keV}$ $\left({ }^{55} \mathrm{Mn} K_{\alpha}\right.$-line) up to $5.5 \mathrm{MeV}\left(\alpha\right.$-line of $\left.{ }^{241} \mathrm{Am}\right)$, see [16] and 17$]$. For this work various running conditions have been tested (d.c. bias) in the range $50-65 \mathrm{mK}$ for the bolometer temperature and $200-800 \mathrm{nV} / \mathrm{keV}$ for its sensitivity. 
The wiring and electronic readout system is flexible and can be dismantled. Emphasis is put on achieving optimal electronic noise (no ground loop, careful shielding), and reduced sensitivity to microphonics. Up to six signal channels can be implemented on this set-up. The preamplifier is designed according to ref.18, with a low-noise FET (enclosed in a $4.2 \mathrm{~K}$ box) heated to 120-150 K. For the highest bolometer temperature, a flat noise spectrum is obtained at a level $\leq 2 \mathrm{nV} \cdot \mathrm{Hz}^{-1 / 2}$, close to the thermodynamic limit. The increase of the detector sensitivity, by lowering the temperature, induces the rise of microphonic peaks in the noise spectrum, preventing any improvement of the energy threshold. An electronic threshold lower than $2 \mathrm{keV}$ and a baseline width (FWHM) around $1 \mathrm{keV}$ are obtained.

The data acquisition chain includes a low-noise amplifier, a low-pass filter $(\leq 1 \mathrm{kHz})$ and a 12 bits Flash-ADC, allowing the event shape recording. Specially developed software allows control and read out of the digitizer, on-line data treatment and storage, remote control of the acquisition.

\subsection{Energy calibration}

Due to the close lead shield around the bolometer and to cryogenic constraints, it is difficult to insert (and remove!) a gamma source close enough to the detector to observe low energy lines. As no line is present in the background spectrum (small bolometer of low $\mathrm{Z}$ material), there is no possibility of self-calibration. Thus a ${ }^{60}$ Co source, providing lines at 1.173 and $1.333 \mathrm{MeV}$, is used for the calibration and set near the detector, inside the outer shield. The Compton spectrum obtained with acquisition time between 0.5 and $1 \mathrm{~h}$ exhibits two well-defined Compton edges at 0.960 and $1.116 \mathrm{MeV}$, allowing an energy calibration with an uncertainty within $5 \%$ (energy binning, detector resolution). 
This calibration holds for lower energies down to a few $\mathrm{keV}$ if the non-linearity of the detector is sufficiently low at this temperature. In order to check the non-linearity, the coldest run, corresponding to a bolometer temperature of $50 \mathrm{mK}$ is considered. The two Compton edges of ${ }^{60} \mathrm{Co}$ at 0.960 and $1.116 \mathrm{MeV}$ are used together with previous data [19] obtained with the same bolometer, at the same temperature, exposed to a ${ }^{241} \mathrm{Am}$ source (gamma line at $60 \mathrm{keV}$, alpha line at $5.49 \mathrm{MeV}$ ). Fig.3 gives the corresponding plot of the pulse height $V$ versus the energy $E$ fitted by the function $V=a E /(1+b E)$. For the $50 \mathrm{mK}$ run, the adjusted value of b yields an overestimate of about $5 \%$ of the energy of $4 \mathrm{keV}$ events, when extrapolating the pulse height of the ${ }^{60}$ Co Compton edges down to low values assuming linearity; the non-linearity decreases for higher temperature runs. It should be noted that this small, not-corrected, non-linearity corresponds to an overestimate of the energy threshold and to a conservative exclusion plot (see section 4).

\section{Background measurements and data analysis}

While the rise and decay times of the pulses obtained during calibration runs with the ${ }^{60} \mathrm{Co}$ source (Compton events) exhibit a low dispersion $(1.5 \%$ at $1 \sigma)$, a significant fraction of the pulses corresponding to background measurements have rise and decay times which differ, up to a factor of two from these mean values (these variations being uncorrelated with the pulse energy). Then, to define a mean "true" radioactive event, only the pulses of the calibration runs are considered; the shape of these pulses has been carefully checked and found independent of the energy in the $20 \mathrm{keV}-1.2 \mathrm{MeV}$ range.

Fig. 4(a-c), presents typical background events superposed on the mean "true" radioactive event (the fitting procedure is described hereafter): 
(a) a standard background event with a shape identical to the mean true radioactive event shape:

(b) a short pulse; it has been experimentally verified that this type of event is due to electromagnetic noise (off/on neon lights, ...);

(c) a long pulse showing a standard rise time but an extended decay tail; this population amounts to about $10 \%$ of the total number of events and is distributed all over the $5-100$ $\mathrm{keV}$ energy region. The physical mechanism leading to this distorted shape is not yet understood. Nevertheless as this population is not present in the high counting rate calibration runs $(\sim 1.5 \mathrm{~Hz})$ but appears only in the low counting background measurements $(\sim$ $0.01 \mathrm{~Hz}$ ), it may be concluded that these long pulses are related to internal contaminations of the device and have to be discarded.

The complete off-line analysis proceeds as follows. Firstly, a template $G(t)$ is built using low energy Compton events from the ${ }^{60}$ Co calibration runs. Each event $S(t)$ of a background run is then fitted to this template, by $\chi^{2}$ minimisation with only two free parameters: starting time of the event $\left(i_{0}\right)$ and pulse height $(\alpha), \chi^{2}=\sum_{i=1}^{N} \frac{\left(S_{i-i_{0}}-\alpha G_{i}\right)^{2}}{\sigma^{2}}$, $\sigma$ being the standard deviation of the baseline noise. The energy of the event is then $\alpha$, normalised to the energy of the template event. Our criterion is to keep as physical signals those with a $\chi^{2}$ per degree of freedom $\chi^{2} / N$ lower than 1.5 , (typically $N \sim 1000$ samples). This cut-off, while retaining more than $99 \%$ of the radioactive background events, provides an efficient elimination of spurious (e.g. electrical and acoustical) events down to $5 \mathrm{keV}$. Below $5 \mathrm{keV}$, the $\chi^{2}$ versus energy scatter plot shows unambiguously that a significant fraction of the low $\chi^{2}$ events $\left(\chi^{2} / N<1.5\right)$ is constituted by non-radioactive noise. As this fraction becomes of the order of $50 \%$ below $4 \mathrm{kel}$. this value is then adopted as the actual 
energy threshold.

In order to reduce the contribution of microphonics an optimal filtering treatment is also performed. Using the same template, the energy of an event is now obtained by $\chi^{2}$ minimisation in the frequency space, $\chi^{2}=\sum_{i=1}^{N} \frac{\left|S_{i-i_{0}}(f)-\alpha G_{i}(f)\right|^{2}}{|N(f)|^{2}}$. The mean noise spectrum $N(f)$ is computed using baselines acquired at the beginning of each run (trigger auto mode). The $\chi^{2}$ cut-off is taken, as before, at $\chi^{2} / N=1.5$. The resulting spectra are in general similar to those obtained from the preceding time space treatment, with the same $4 \mathrm{keV}$ threshold.This procedure is found more efficient only for the high sensitivity runs, showing rather strong microphonic peaks in their noise spectra : the content of the first 4-6 $\mathrm{keV}$ bin is reduced by a factor of $\sim 2$.

The sum of all the runs yields the background spectrum given on fig.5, corresponding to a running time of 160 hours. In the energy range above $16 \mathrm{keV}$, a rather flat spectrum is measured, with a mean event rate of 25 events. $\mathrm{keV}^{-1} \cdot \mathrm{kg}^{-1} \cdot \mathrm{day}^{-1}$. This value is ten times below the rate obtained in a previous attempt performed with the same bolometer [17] and a factor $\sim 4$ higher than the background measured in the NaI scintillator experiment [20]. Below $16 \mathrm{keV}$, the event rate grows steeply.

Additional low-level radioactivity measurements are in progress, in order to understand the origin of this background. The low energy event rate is clearly enhanced by the lack of containment of our small size detector: $\gamma$ rays of typically $1 \mathrm{MeV}$, non absorbed in the crystal, are degraded in lower energy events by Compton scattering.

4. Limits on WIMPs scattering cross-sections

Cross section versus WIMP mass exclusion plots are derived through the procedure given, 
for instance, in ref.21; this allows a fully significant comparison between the results of different experiments.

For a given WIMP mass, the expected recoil energy spectrum is calculated [22], taking a scattering cross-section equal to unity; it is then folded with the instrumental response function. In this calculation a nuclear form factor is included following Engel [23] in the case of spin-independent interactions. In the spin-dependent case, the same shape of form factor is taken using the values of the ratio of the spin radius over the charge radius given by Ellis and Flores [24]. As expected for light nuclei, the influence of these form factors on the calculated spectrum is negligible. The lowest cross-section to which the experiment is sensitive is searched by dividing, in each energy bin, the experimental spectrum at $95 \%$ CL (the measured spectrum increased by $2 \sigma$ ) by the theoretical one.

The following parameters for the Galactic halo model of WIMPs are used: local density $0.3 \mathrm{GeV} / \mathrm{cm}^{3}$, Earth velocity in the Galactic frame $220 \mathrm{~km} / \mathrm{s}$ (data taken between November and January), WIMP's Maxwellian distribution velocity $270 \mathrm{~km} / \mathrm{s}$ r.m.s., cut-off at an escape velocity of $800 \mathrm{~km} / \mathrm{s}$.

The experimental parameters used are: an energy threshold of $4 \mathrm{keV}$, as discussed in section 3; a response function of Gaussian shape with $\sigma=1 \mathrm{keV}$, constant in the 4 $100 \mathrm{keV}$ range, corresponding to the mean baseline width. WIMP interactions produce nuclear recoils and the detector is calibrated using gamma sources. Then the "quenching factor function" $\mathrm{Q}\left(\mathrm{E}_{r}\right)=\mathrm{S}_{\text {nucleus }}\left(\mathrm{E}_{r}\right) / \mathrm{S}_{\text {gamma }}\left(\mathrm{E}_{r}\right)$ (S being the signal amplitude) is needed to analyse the spectrum. $\mathrm{Q}\left(\mathrm{E}_{\tau}\right)$ has been measured at neutron beams $[25,26)$ to range from 0.07 to 0.3 for ionisation and scintillation detectors at energies of the order of a few keV. Enfortunately such measurements turn out to be very difficult with a slow bolometer, 
as the one used in this experiment. Two groups, using the ${ }^{206} \mathrm{~Pb}$ recoil nucleus released after the $\alpha$-decay of ${ }^{210} \mathrm{Po}$, measured $\mathrm{Q}\left(\mathrm{E}_{r}\right)$ at $100 \mathrm{keV}$. They found $0.98 \pm 0.05([27]$, diamond bolometer $)$ and $0.93 \pm 0.03$ ([28], $\mathrm{TeO}_{2}$ bolometer). At lower energies no direct measurement is available, but intensive studies of solids under irradiation [29] and associated numerical computations in sapphire showed that less than $5 \%$ of the recoil energy is trapped in the lattice's Frenkel defects generated by the recoiling nuclei down to a few hundred $\mathrm{eV}$. Thus the quenching factor may be assumed with confidence as greater than 0.95 , and given the precision of this experiment, it has been assumed equal to 1 in the following analysis.

Fig.6 shows the exclusion plot for WIMPs interacting with nuclei through neutrinolike vector coupling; both ${ }^{27} \mathrm{Al}$ and ${ }^{16} \mathrm{O}$ nuclei contribute to the recoil spectrum. The WIMP scattering cross-section on a nucleus, $\sigma_{\mathrm{WN}}$, is $[12,30]$

$$
\sigma_{\mathrm{WN}}=\left(\frac{\mu_{\mathrm{WN}}}{\mu_{\mathrm{W}_{\mathrm{n}}}}\right)^{2} \bar{N}^{2} \sigma_{\mathrm{Wn}},
$$

where $\mu_{W_{N}}$ and $\mu_{W_{n}}$ are the reduced masses of the WIMP-nucleus and WIMP-neutron systems, $\bar{N}=(A-Z)-\left(1-4 \sin ^{2} \theta_{W}\right) Z \approx A-Z$ the number of neutrons in nucleus and $\sigma_{\mathrm{Wn}}$ the WIMP cross section on the neutron.

To compare the results obtained for various nuclei, it is convenient to present the exclusion plots in terms of $\sigma_{W_{n}}$. The other experimental data shown on fig.6 are taken from the literature and correspond to a given nucleus; they are renormalised to $\sigma w_{n}$. It can be noted that, due to the low value of $\bar{N}^{2}$ for the ${ }^{2 i} \mathrm{Al}$ and ${ }^{16} \mathrm{O}$ nuclei, the sapphire is not favoured for this type of interaction.

Fig.7 stands for axially coupled particles interacting with non-zero spin nuclei. The cross- 
section is now $[12,24]$, for an odd-type nucleus with an unpaired proton,

$$
\sigma_{\mathrm{WN}}=\frac{\mu_{\mathrm{WN}}^{2}\left[\lambda^{2} J(J+1)\right]_{\text {Nucleus }}}{\mu_{\mathrm{W}_{\mathrm{p}}}^{2}\left[\lambda^{2} J(J+1)\right]_{\mathrm{proton}}} \sigma_{\mathrm{W}_{\mathrm{F}}}
$$

$\mu_{\mathrm{WN}}$ and $\mu_{\mathrm{W}}$ are the reduced masses of the WIMP-nucleus and WIMP-proton systems, $\lambda^{2} J(J+1)$ the spin nuclear matrix element of the nucleus (values taken from ref.31, ${ }^{1} \mathrm{H}$ : $\left.0.75,{ }^{27} \mathrm{Al}: 0.087\right)$ and $\sigma_{\mathrm{W}_{\mathrm{p}}}$ the WIMP scattering cross-section on a proton for which the limit cross-section is given on fig.7.

Comparison is made on fig.7 with scintillating crystal experiments ( $\mathrm{NaI},[20]$ and $\mathrm{CaF}_{2}$, [21]). The difference in sensitivity between the various experiments is explained by the combined effects of the achieved background rates and of the spin matrix elements of the nuclei involved. For ${ }^{23} \mathrm{Na}$ the spin factor value $(0.041,[31])$, less favourable than for ${ }^{27} \mathrm{Al}$, is balanced by the lower counting rates. On the contrary, the very high spin factor of ${ }^{19} \mathrm{~F}$ $(0.647,[31])$ leads to a better sensitivity of the $\mathrm{CaF}_{2}$ experiment [21], despite a background at the same level as the one measured in the present work.

\section{Conclusion}

The EDELWEISS bolometric detection facility is now operational, as shown by the present results. Work in progress includes an upgrade of the dilution refrigerator (lower temperature, larger experimental volume) and a specific effort to reduce the radioactive background arising from both the dilution refrigerator and the detectors. To increase the sensitivity to WIMP's scattering we are preparing new experiments with a $80 \mathrm{~g}$ Ge bolometer (with both thermal and ionisation signals) and with sapphire bolometers of larger mass (thermal signal only). 


\section{Acknowledgments}

It is a pleasure to thank the members of the LSM staff for their efficient help as well as F. and Ph. Hubert (CENBG), L. Mosca (DAPNIA) and J.L. Reyss (CFR) for the lowradioactivity measurements of the experiment components. M. Martin (IAP), L. Bergé, M. Capel, D. Carré and G. Gravant (CSNSM) are gratefully acknowledged for their technical and efficient support.

Special thanks are due to M. L'Hour (Direction des Recherches Archéologiques SousMarines) and to Direction du Patrimoine, Ministère de la Culture, for the bestowal of archaeological lead.

This work has been supported by CNRS (IN2P3, INSU, Ultimatech) and CEA/DSM.

\section{References}

[1] C. Alcock et al., Nature 365 (1993) 621

[2] E. Aubourg et al., Nature 365 (1993) 623

[3] A. Udalski et al., Astrophys. J. L69 (1994) 426

[4] R. Ansari et al., Nucl. Phys. B (proc. suppl.) 43 (1995) 165

[5] C. Alcock et al., Phys. Rev. Lett. 74 (1995) 2867

[6] E.I. Gates , G. Gyuk and M.S. Turner, Phys. Rev. Lett. 74 (1995) 3724

[7] A. Songaila, L.L. Cowie, C.J. Hogan and M. Rugers, Nature 368 (1994) 599

8] R. Carswell, M. Rauch, R. Weymann, A. Cooke and J.K. Webb, Month. Not. R. Astron. Soc. 268 (1994) L1

9] E. Vangioni-Flam and M. Cassé, Astrophys. J. 441 (1995) 471

10: G.B. Gelmini, P. Gondolo and E. Roulet, Nucl. Phys. B 351 (1991) 623 
A. Bottino, V. de Alfaro, N. Fornengo, G. Mignola and M. Pignone, Astrop. Phys. 2 (1994) 67

[12] M.W. Goodman and E. Witten, Phys. Rev. D 31 (1985) 3059

[13] L. Mosca, in "Particle Astrophysics, Atomic Physics and Gravitation", eds. J. Tran

Thanh Van, G. Fontaine and E. Hinds (Editions Frontières, Gif-sur-Yvette, 1994)

[14] Proceedings of the 6th Intern. Workshop on "Low Temperature Detectors", Interlaken,1995, eds H.R. Ott and A. Zehnder, to be published in Nucl. Instr. Meth. A

[15] V. Chazal et al., private communication

[16] P. de Marcillac et al.,"Low Temperature Detectors for Neutrinos and Dark Matter IV", eds N.E. Booth and G.L. Salmon (Editions Frontières, Gif-sur-Yvette, 1992)

[17] N. Coron et al., Astron. Astrophys. 278 (1993) L31

[18] D. Yvon et al., accepted in Nucl. Instr. Meth. A

[19] P. de Marcillac, private communication

[20] C. Bacci et al., Phys. Lett. B 293 (1992) 460

[21] C. Bacci et al., Astrop. Phys. 2 (1994) 117

[22] P.F. Smith and J.D. Lewin, Physics Reports 187 (1990) 203

[23] J. Engel, Phys. Lett. B 264 (1991) 114

[24] J. Ellis and R.A. Flores, Phys. Lett. B 263 (1991) 259

[25] C. Chasman et al., Phys. Rev. Lett. 21 (1968) 1430 and references therein; G. Gerbier et al., Phys. Rev. D42 (1990) 3211; A.R. Sattler, F.L. Vook and J.M. Palms, Phys. Rev. $143(1966) 588$

[26] T. Shutt et al., Phys. Rev. Lett. 69 (1992) 24; N. Spooner et al., Phys. Lett. B 273 (1991) 333; B.L. Dougherty et al., J. Low Temp. Phys. 93 (1993) 365 
j.W. Zhou et al., Nucl. Instr. Meth. A 349 (1994) 225 and references therein [28] Milano group, private communication

[29] M.T. Robinson, Phil. Mag. 17 (1968) 639; D. Lesueur, Phil. Mag. 44 (1981) 905 and private communication

[30] J.R. Primack, D. Seckel and B. Sadoulet, Ann. Rev. Nucl. Part. Sci. 38 (1988) 751

[31] J. Engel and P. Vogel, Phys. Rev. D 40 (1989) 3132

[32] D.O. Caldwell et al., Phys. Rev. Lett. 65 (1990) 1305

[33] D. Reusser et al., Phys. Lett. B 255 (1991), 143 


\section{ecaptions}

fig.1. General drawing of the EDELWEISS set-up.

Fig.2. Schematic diagram of the lower part of the dilution unit showing the shielding of the experimental chamber.

Fig.3. Energy calibration curve of the $24 \mathrm{~g}$ sapphire bolometer at $50 \mathrm{mK}$.

Fig.4. Three typical background events (run of Dec 20-22 1994, $41.4 \mathrm{~h}$ ). The quoted energy is derived from the pulse height.

Fig.5. Measured event rate for the $24 \mathrm{~g}$ sapphire bolometer. The quoted errors are statistical ( 1 sigma).

Fig.6. Exclusion plots for vector coupled WIMPs, normalised to the WIMP-neutron crosssection. $\mathrm{Al}_{2} \mathrm{O}_{3}(94)$ : this work. $\mathrm{Al}_{2} \mathrm{O}_{3}(91)$ : $24 \mathrm{~g}$ sapphire bolometer previous experiment [17]. NaI: NaI scintillator experiment [20], the ${ }^{127} \mathrm{I}$ nucleus gives lower limiting crosssections for WIMP's masses greater than $\sim 40 \mathrm{GeV}$. Si: silicon diode experiment [32]. Ge: germanium diode experiment [33].

Fig.7. Exclusion plots for axially coupled WIMPs, normalised to the WIMP-proton crosssection. Al(94): this work. Al(91): 24g sapphire bolometer previous experiment [17]. $\mathrm{Na:}$ NaI scintillator experiment [20]. F: $\mathrm{CaF}_{2}$ scintillator experiment $[21]$. 


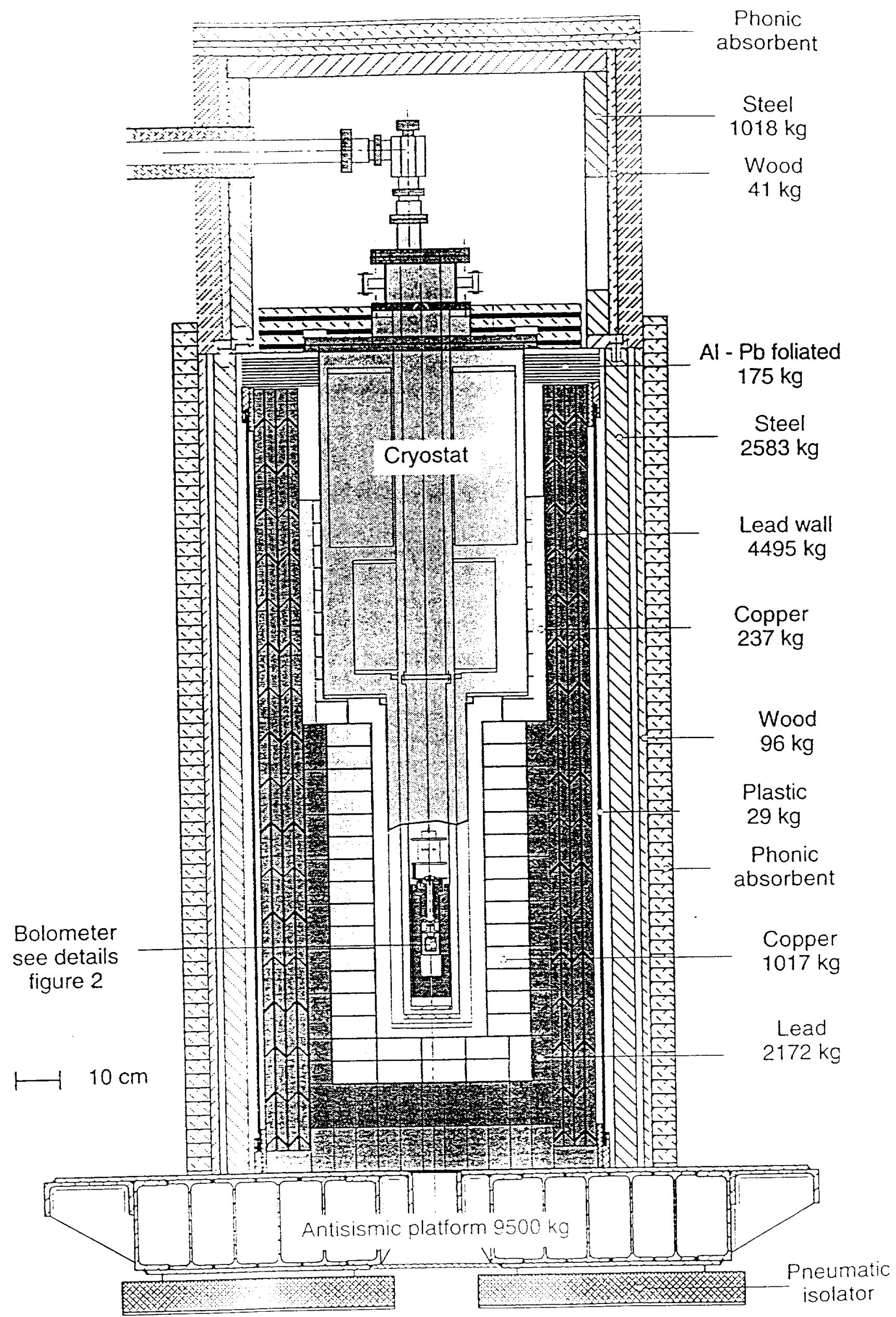




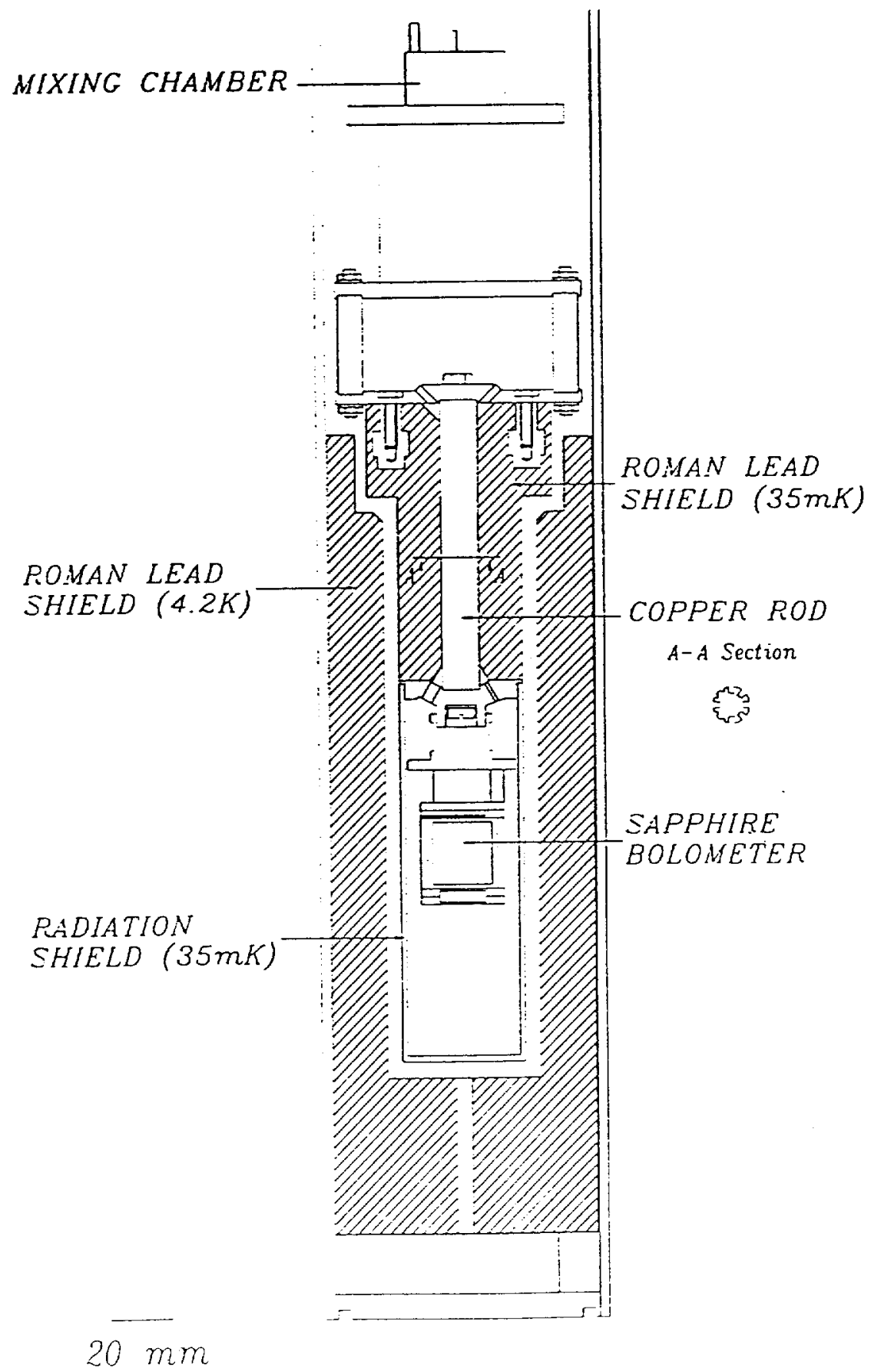




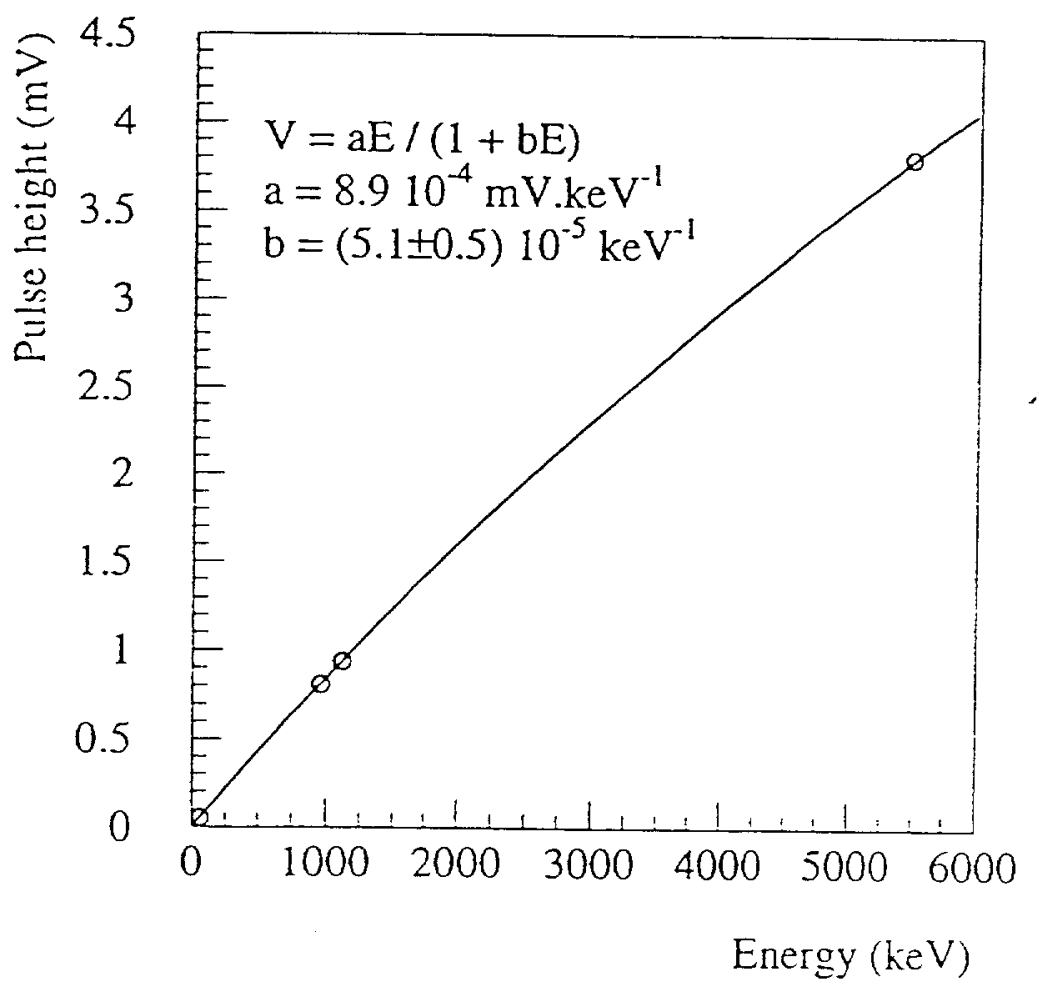

Fig 3 

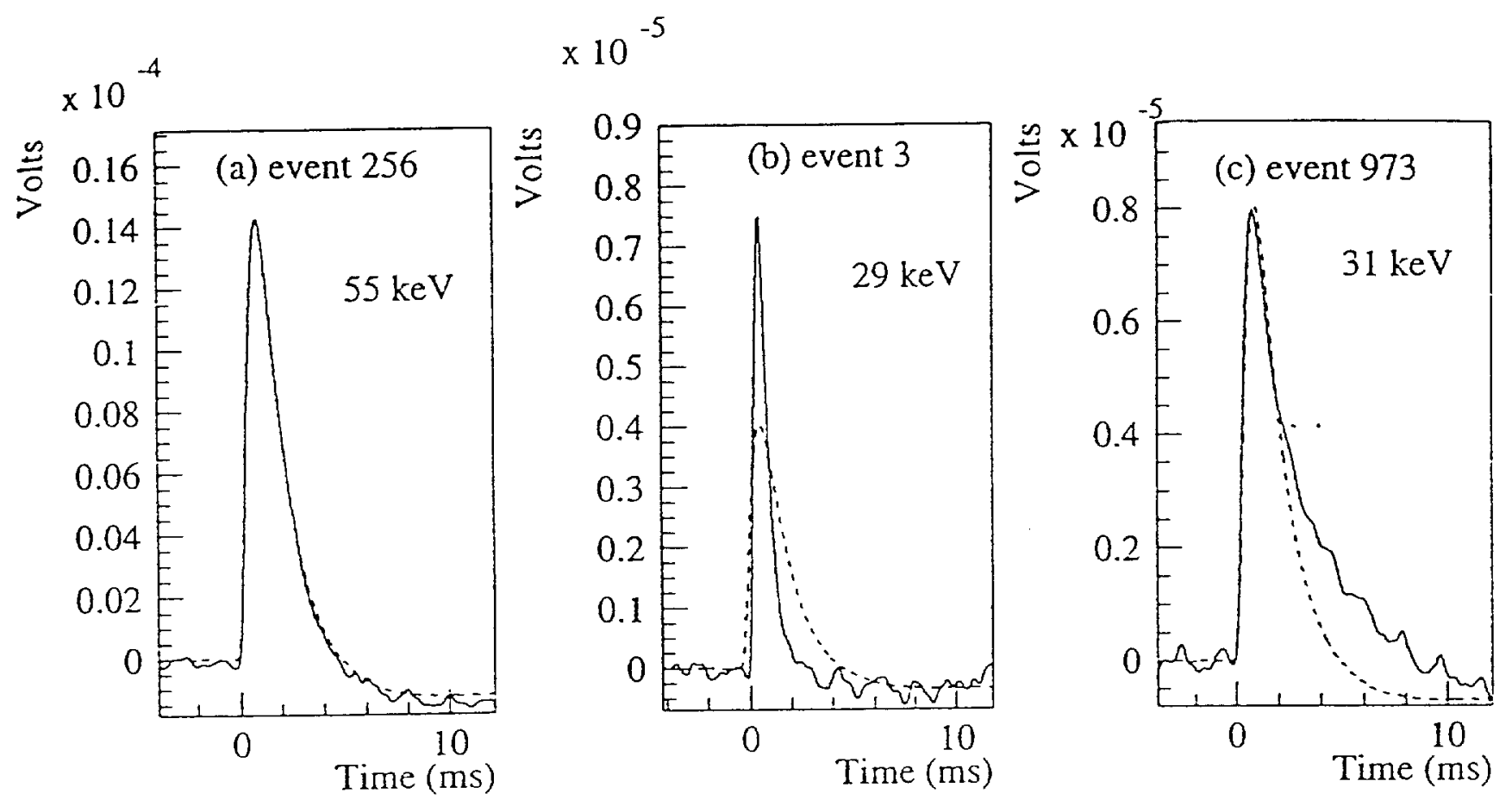

Fig 4 


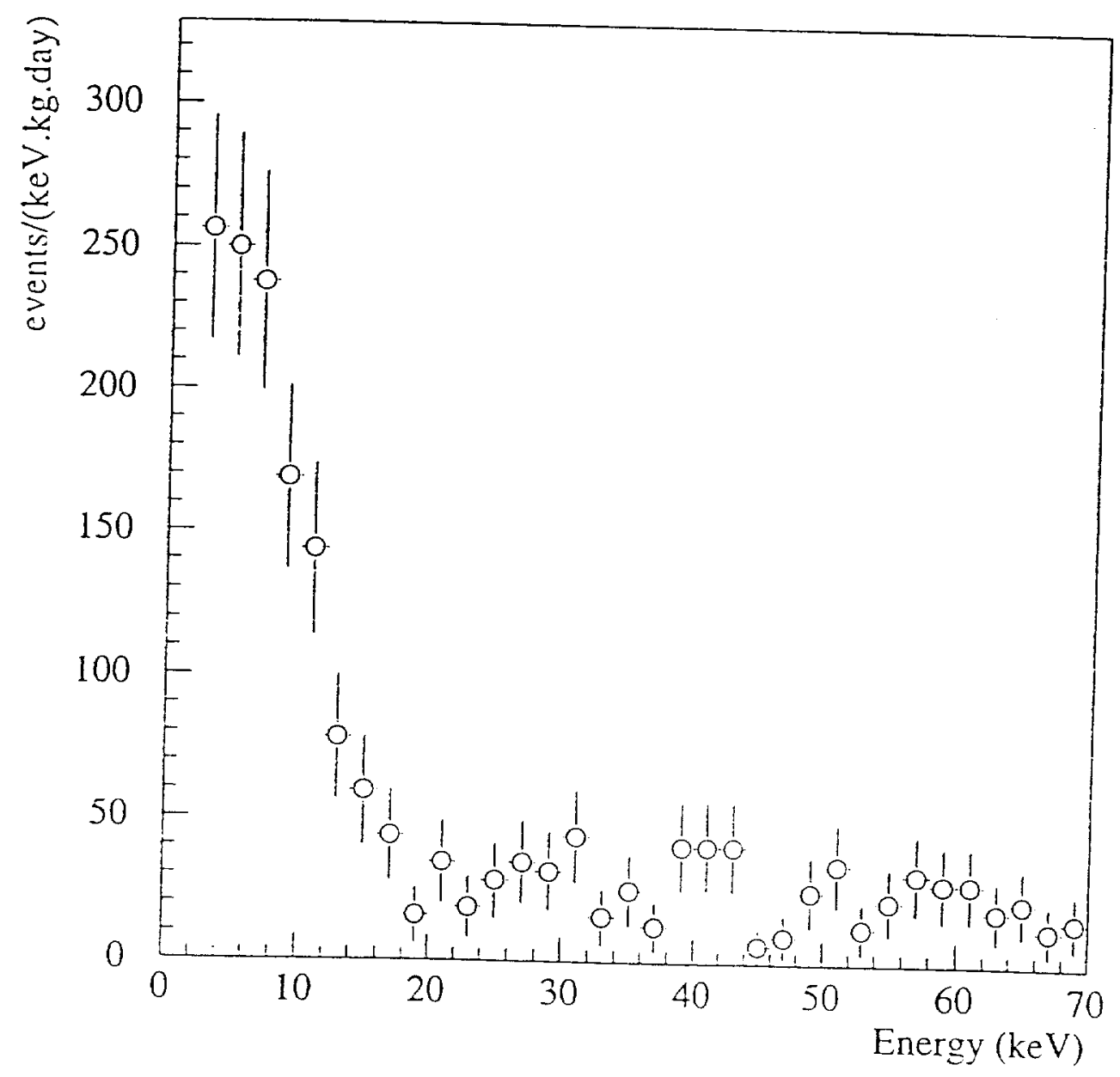

Fig 5 


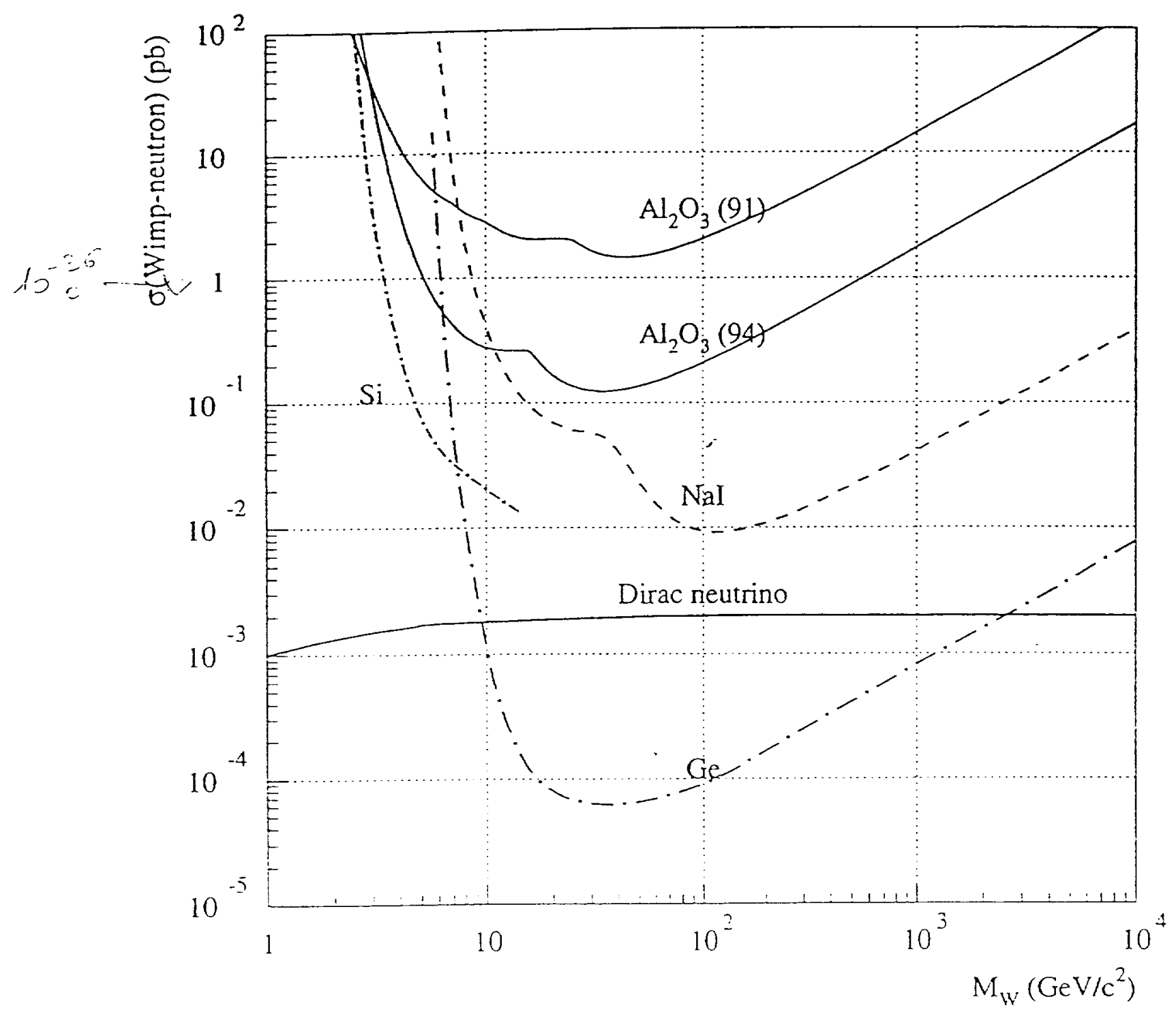

Fig 6 


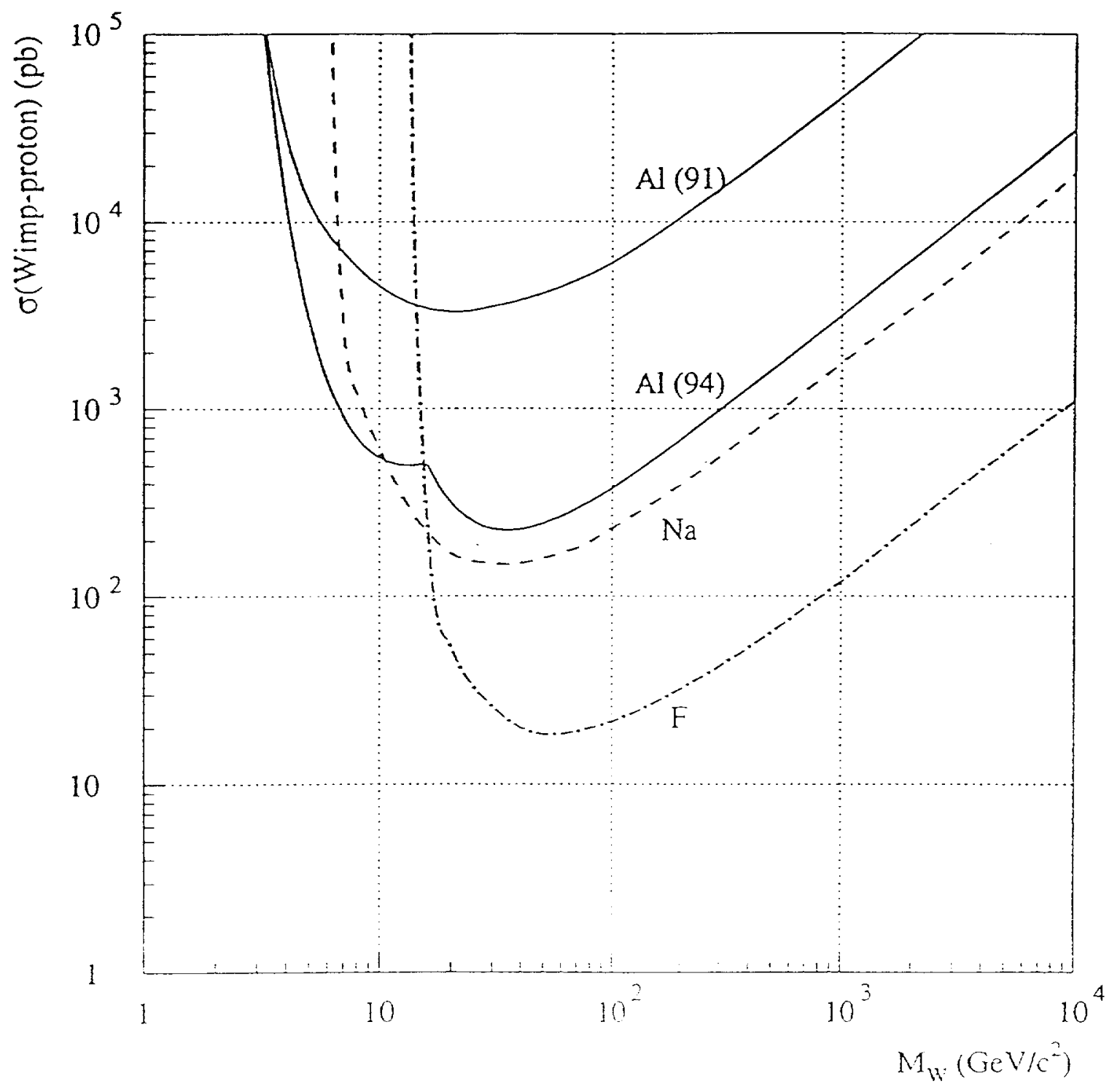

Fig 7 
